\title{
Consensus for wireless sensor networks with Markovian switching topology and stochastic communication noises
}

\author{
Wuneng Zhou*, Chuan Ji', Jinping Mou, Anding Dai and Jian'an Fang
}

\author{
"Correspondence: \\ zhouwuneng@163.com; \\ jichuan@mail.dhu.edu.cn \\ College of Information Sciences and \\ Technology, Donghua University, \\ Shanghai, 200051, China
}

\begin{abstract}
In this paper, we consider the problem of average consensus for wireless sensor networks (WSNs). By applying the Lyapunov function and the M-matrix approach, several sufficient conditions to ensure the average consensus in $p$ th moment for WSNs with Markovian switching topology are derived. Also we study the stochastic average consensus in the large for WSNs with Markovian switching topology, whose transition jump rates matrix is not precisely known. We finally show the numerical simulations to illustrate the effectiveness of the results derived in this paper.

Keywords: wireless sensor networks; consensus in pth moment; Markovian switching topology; stochastic communication noises; stochastic average consensus in the large
\end{abstract}

\section{Introduction}

With the increasing development of microelectronics, wireless communication and computing, wireless sensor networks (WSNs) have been more widely used in such areas as robotics, surveillance, environment monitoring, information collection, and so on.

From a network-theoretic perspective, a WSN can be looked upon as a multi-agent system (MAS) with each node representing a sensor and each edge performing information exchange between sensors. In some cases, the agreement is a common value which may be the average of the initial states of the system, and which is often called average consensus and has wide application background in the areas such as formation control [1], distributed filtering [2] and distributed computation [3]. It means to achieve the accordance of the states of MAS. In [4], the authors considered the average consensus control for the directed and undirected networks with fixed and switching topologies. In [5], the authors extended the results of [4] to the discrete-time models and weakened the condition of instantaneous strong connectivity. In [6], the authors considered the distributed averaging consensus of the networks with fixed and undirected topologies.

In the real communication surroundings, the topology of the WSN will change among some modes along with the time. Due to the limited energy, the sensor nodes often switch the states between sleep and awake to save energy. So we make use of Markov switching topology to describe this situation. Zhang [7] designed a distributed consensus protocol to analyze the multi-agent systems in uncertain communication environments including the communication noises and Markov topology switches. The asymptotic unbiased mean

\section{Springer}

C2013 Zhou et al: licensee Springer. This is an Open Access article distributed under the terms of the Creative Commons Attribution License (http://creativecommons.org/licenses/by/2.0), which permits unrestricted use, distribution, and reproduction in any medium, provided the original work is properly cited. 
square average consensus and asymptotic unbiased almost sure average consensus were obtained. Also they considered the effect of agent dependent gain functions on the consensus of multi-agent systems. Zhou et al. [8] studied the problem of the mode and delaydependent adaptive exponential synchronization in $p$ th moment for stochastic delayed neural networks with Markovian switching. By applying a novel nonnegative function and the $M$-matrix approach, several sufficient conditions to ensure the mode and delay dependent adaptive exponential synchronization in $p$ th moment for stochastic delayed neural networks were derived. Zhou et al. [9] considered the problem of target synchronization for the WSNs with multiple time-varying delays, Markov switching topology and the stochastic disturbance. The communication topology graphs they studied in this paper were undirected.

In real networked control systems, the packet dropouts and channel delays usually arise in the communication among the sensor nodes. The situations can be modeled by the Markov chain with the assumption of completely known or incompletely known transition probabilities. Therefore, from control perspectives, it is important and necessary to discuss the systems with partly unknown transition probabilities. Zhang and Boukas [10] studied the stability and stabilization problems of a class of continuous-time and discretetime Markovian jump linear systems with partly unknown transition probabilities. Zhang and Lam [11] considered the Markov jump linear system with incomplete transition descriptions and got the necessary and sufficient criteria in both continuous-time domain and discrete-time cases. Zhu et al. [12] discussed the asymptotic stability of nonlinear stochastic differential equations with Markovian switching. The transition jump rates matrix $\Gamma$ is not precisely known for the equations.

Moreover, to study the average consensus for the WSNs with Markovian switching topology and stochastic noises, we take advantage of the $p$ th moment exponential stability theory [13] which is widely applied to the system stability analysis [14-16]. Specially, the case $p=2$ is the mean square exponential stability which is frequently used in different research works such as [17-19]. In [19], the authors showed that a necessary and sufficient condition for asymptotically unbiased mean square average consensus is that the consensus gains satisfy the step rule similar to that of classical stochastic approximation.

Next, we introduce our main contributions. To the best of our knowledge, the method combining the Lyapunov function and the $M$-matrix is rarely used in the researching area of the average consensus in $p$ th moment for WSNs with Markovian switching topology and stochastic noises. Also, the stochastic average consensus in the large for WSNs with Markovian switching topology, whose transition jump rates matrix is not precisely known, is rarely discussed. The present paper considers the consensus in $p$ th moment for WSNs with Markovian switching topology and stochastic noises in communication. Making use of the $p$ th moment exponential stability theory, we propose a sufficient condition of the $p$ th moment exponential consensus for the WSNs. Different from [9], the communication topology graphs discussed are directed. We also consider the stochastic average consensus in the large for the WSN, whose transition jump rates matrix $\Gamma$ is not precisely known.

The remainder of the paper is organized as follows. In Section 2, some concepts in graph theory are described, and the problem to be investigated is formulated. In Section 3, the main results are presented. In Section 4, two numerical examples show the reliability of the main results. In Section 5, some conclusions are given. 
The following notations will be used throughout this paper : $|x|$ represents the Euclidean norm of a vector $x$ in $\mathbb{R}^{n},|A|:=\sqrt{\operatorname{trace}\left(A^{T} A\right)}$, i.e., the trace norm of a matrix $A$. 'T' represents the transpose of a matrix or a vector, $I$ is an $n \times n$ dimensional identity matrix, $1_{n}$ is an $n$-dimensional column vector with all ones, $\mathbb{E}$ stands for the mathematical expectation operator. If $A$ is a matrix, $\lambda_{\min }(A)$ and $\lambda_{\max }(A)$ denote the smallest and largest eigenvalues of $A$, respectively.

\section{Problem formulation and preliminaries}

\subsection{Concepts in graph theory}

Let $\mathcal{G}=\{\mathcal{V}, \mathcal{E}, \mathcal{A}\}$ be a weighted digraph, where $\mathcal{V}=\{1,2, \ldots, n\}$ is the set of nodes, node $i$ represents the $i$ th sensor node, $\mathcal{E}$ is the set of edges, and an edge in $\mathcal{G}$ is denoted by an ordered pair $(j, i) .(j, i) \in \mathcal{E}$ if and only if the $j$ th sensor node can send information to the $i$ th sensor node directly. The neighborhood of the $i$ th sensor node is denoted by $N_{i}=\{j \in$ $\mathcal{V} \mid(j, i) \in \mathcal{E}\}$

$\mathcal{A}=\left[a_{i j}\right] \in \mathbb{R}^{n \times n}$ is called the weighted adjacency matrix of $\mathcal{G}$. For any $i, j \in V, a_{i j} \geq$ 0 , and $a_{i j}>0 \Leftrightarrow j \in N_{i}$. $\operatorname{deg}_{i n}(i)=\sum_{j=1}^{n} a_{i j}$ is called the in-degree of $i$; $\operatorname{deg}_{\text {out }}(i)=\sum_{j=1}^{n} a_{i j}$ is called the out-degree of $i ; \mathcal{L}=\mathcal{D}-\mathcal{A}$ is called the Laplacian matrix of $\mathcal{G}$, where $\mathcal{D}=$ $\operatorname{diag}\left(\operatorname{deg}_{i n}(1), \ldots, \operatorname{deg}_{i n}(n)\right)$.

\subsection{Average consensus for WSNs}

In this paper, we study the average consensus control for a WSN with dynamics

$$
\dot{x}_{i}(t)=u_{i}(t)
$$

where $x_{i}(t) \in \mathbb{R}$ is the state of the $i$ th sensor, and $u_{i}(t) \in \mathbb{R}$ is the control input. The initial state $x_{i}(0)$ is deterministic.

The $i$ th sensor can receive information from its neighbors

$$
y_{j i}(t)=x_{j}(t)+\sigma_{j i}\left(x_{i}(t), t\right) \omega_{j i}(t)
$$

where $y_{j i}(t)$ denotes the measurement of the $j$ th sensor's state $x_{j}(t)$ by the $i$ th sensors, $\left\{\omega_{j i}(t) \mid i, j=1,2, \ldots, n\right\}$ are the communication noises, $\sigma_{j i}\left(x_{i}(t), t\right)$ is the noise intensity function.

Denote $X(t)=\left(x_{1}(t), x_{2}(t), \ldots, x_{n}(t)\right)^{T},(\mathcal{G}, X)$ is called a dynamic network.

For the dynamic network $(\mathcal{G}, X)$, we use the distributed protocol

$$
u_{i}(t)=\sum_{j \in N_{i}} a_{i j}(t)\left(y_{j i}(t)-x_{i}(t)\right) .
$$

Substituting protocol (3) into system (1) leads to

$$
d X(t)=-L(t) X(t) d t+G(X(t), t) d W(t)
$$

where $L(t)$ is the Laplacian matrix of $\mathcal{G}, G(X(t), t)$ is the noise intensity matrix, $W(t)=$ $\left(W_{1}(t), \ldots, W_{n}(t)\right)^{T}$ is an $n$-dimensional Brownian motion.

In WSNs, each sensor node communicates with other sensor nodes through the unreliable networks. If the communication channel between sensors $i$ and $j$ is $(j, i) \in \mathcal{E}_{f}, \mathcal{E}_{f}$ is the 
set of the communication channel which probably lost the signal, then the time-varying topologies under link failure or creation can be described by Markov switching topology. Let $\left\{r(t)_{t \geq 0}\right\}$ be a right-continuous Markovian chain on the probability space taking values in a finite state set $S=\{1,2, \ldots, N\}$ with generator $\Gamma=\left(\gamma_{i j}\right)_{N \times N}$ given by

$$
P\{r(t+\Delta)=j \mid r(t)=i\}= \begin{cases}\gamma_{i j} \Delta+o(\Delta) & \text { if } i \neq j \\ 1+\gamma_{i i} \Delta+o(\Delta) & \text { if } i=j\end{cases}
$$

where $\Delta>0$ and $\gamma_{i j} \geq 0$ is the transition rate from $i$ to $j$ if $i \neq j$ while

$$
\gamma_{i i}=-\sum_{j \neq i} \gamma_{i j}
$$

and $r(0)=r_{0}$.

We denote the directed communication graph by $g=\{\mathcal{G}(1), \mathcal{G}(2), \ldots, \mathcal{G}(N)\}$, where $\mathcal{G}(k)=\left\{\mathcal{V}, \mathcal{E}_{\mathcal{G}(k)}, \mathcal{A}_{\mathcal{G}(k)}\right\}$ is the weighted digraph. Denote the topology graph as $\mathcal{G}_{t}$ at moment $t(t \geq 0)$, so $r(t)=k$ if and only if $\mathcal{G}_{t}=\mathcal{G}(k)$.

Under Markovian switching topology, we have

$$
d X(t)=-L(r(t)) X(t) d t+G(X(t), r(t)) d W(t) .
$$

Next, we consider the average consensus control protocol for system (6) as follows:

$$
d \delta(t)=-L(r(t)) \delta(t) d t+(I-J) G(\delta(t), r(t)) d W(t)
$$

where $\delta(t)=X(t)-J X(t), J=\frac{1}{n} 1_{n} 1_{n}^{T}$.

If we define

$$
f(\delta, t, i)=-L(r(t)) \delta(t), \quad g(\delta, t, i)=(I-J) G(\delta(t), r(t)),
$$

then (7) becomes

$$
d \delta(t)=f(\delta, t, i) d t+g(\delta, t, i) d W(t) .
$$

Definition 1 Dynamic system (6) is said to be average consensus in $p$ th moment if error system (7) satisfies that $\limsup _{t \rightarrow \infty} E|\delta(t)|^{p}=0$. If $p=2$, we called it reach mean-square average consensus.

Definition 2 Dynamic system (6) is said to reach stochastic average consensus in the large if error system (7) is stochastic stable and, moreover,

$$
\mathbb{P}\left\{\lim _{t \rightarrow \infty} \delta\left(t ; t_{0}, \delta_{0}, i\right)=0\right\}=1 .
$$

To get the main results, we need the following assumptions.

(A1) $\mathcal{G}$ is a balanced digraph;

(A2) The union of $\{\mathcal{G}(i) \mid i \in S\}$ contains a spanning tree. 
(A3) There exists a positive constant $H$ such that the noise intensity matrix $g(\cdot, \cdot, \cdot)$ satisfies

$$
\operatorname{trace}\left(g^{T}(\delta, t, i) g(\delta, t, i)\right) \leq H|\delta(t)|^{2} .
$$

To this end, we introduce some concepts and lemmas which will be used in the proofs of our main results.

Consider an $n$-dimensional stochastic differential equation with Markovian switching

$$
d x(t)=\tilde{f}\left(x(t), r_{t}, t\right) d t+\tilde{g}\left(x(t), r_{t}, t\right) d W(t)
$$

on $t \in[0, \infty)$ with the initial data $x\left(t_{0}\right)=x_{0} \in L_{\mathcal{L}_{0}}^{p}\left(\Omega ; R^{n}\right)$ and $r\left(t_{0}\right)=r_{0}$.

For $V \in C^{2,1}\left(R^{n} \times S \times R_{+} ; R_{+}\right)$, define an operator $\mathcal{L}$ from $R^{n} \times S \times R_{+}$to $R$ by

$$
\begin{aligned}
\mathcal{L} V(x(t), i, t)= & V_{t}(x(t), i, t)+V_{x}(x(t), i, t) \tilde{f}(x(t), i, t) \\
& +\frac{1}{2} \operatorname{trace}\left(\tilde{g}^{T}(x(t), i, t) V_{x x}(x(t) i, t) \tilde{g}(x(t), i, t)\right) \\
& +\sum_{j=1}^{N} \gamma_{i j} V(x(t), j, t),
\end{aligned}
$$

where

$$
\begin{aligned}
& V_{t}(x(t), i, t)=\frac{\partial V(x(t), i, t)}{\partial t}, \\
& V_{x}(x(t), i, t)=\left(\frac{\partial V(x(t), i, t)}{\partial x_{1}}, \frac{\partial V(x(t), i, t)}{\partial x_{2}}, \ldots, \frac{\partial V(x(t), i, t)}{\partial x_{n}}\right), \\
& V_{x x}(x(t), i, t)=\left(\frac{\partial^{2} V(x(t), i, t)}{\partial x_{j} \partial x_{k}}\right)_{n \times n} .
\end{aligned}
$$

Lemma 1 [13] Let $V \in C^{2,1}\left(R^{n} \times R_{+} \times S, R_{+}\right)$and $\tau_{1}$, $\tau_{2}$ be bounded stopping times such that $0 \leq \tau_{1} \leq \tau_{2}$ a.s. If $V(x(t), t, r(t))$ and $\mathscr{L} V(x(t), t, r(t))$ etc., are bounded on $t \in\left[\tau_{1}, \tau_{2}\right]$ with probability 1 , then

$$
\begin{aligned}
\mathbb{E} V\left(\tau_{2}, r\left(\tau_{2}\right), x\left(\tau_{2}\right)\right)= & \mathbb{E} V\left(\tau_{1}, r\left(\tau_{1}\right), x\left(\tau_{1}\right)\right) \\
& +\mathbb{E} \int_{\tau_{1}}^{\tau_{2}} \mathcal{L} V(s, r(s), x(s)) d s .
\end{aligned}
$$

Lemma 2 [20] Let $N_{r}(t)$ be the number of switches on the interval $\left(t_{0}, t\right]$, then we have

$$
P\left(N_{r}(t)=k\right) \leq e^{-\tilde{q} t} \frac{(\bar{q} t)^{k}}{k !}, \quad \forall k \in \mathbb{N},
$$

where $\tilde{q}=\max \left\{\gamma_{i j}: i, j \in S\right\}, \bar{q}=\max \left\{\left|\gamma_{i i}\right|: i \in S\right\}$.

Definition 3 [21] A square matrix $M=\left(m_{i j}\right)_{n \times n}$ is called a nonsingular $M$-matrix if $M$ can be expressed in the form of $M=s I_{n}-G$ with some $G \geq 0$ (i.e., each element of $G$ is nonnegative) and $s>\rho(G)$, where $\rho(G)$ is the spectral radius of $G$. 
Lemma 3 [13] If $M=\left(m_{i j}\right)_{n \times n} \in R^{n \times n}$ with $m_{i j}<0(i \neq j)$, then the following statements are equivalent:

(1) $M$ is a nonsingular M-matrix;

(2) Every real eigenvalue of $M$ is positive;

(3) $M$ is positive stable. That is, $M^{-1}$ exists and $M^{-1}>0$ (i.e., $M^{-1} \geq 0$ and at least one element of $M^{-1}$ is positive).

Lemma 4 [13] (Young inequality) Let $a, b \in R$ and $\beta \in[0,1]$. Then

$$
|a|^{\beta}|b|^{(1-\beta)} \leq \beta|a|+(1-\beta)|b| .
$$

Lemma 5 [13] (Gronwall inequality) Let $T>0$ and $u(\cdot)$ be a Borel measurable bounder nonnegative function on $[0, T]$. If

$$
u(t) \leq \alpha(t)+\int_{0}^{t} \beta u(s) d s, \quad \forall 0 \leq t \leq T
$$

for the non-decreasing function $\alpha(t)$ and the constant $\beta$, then

$$
u(t) \leq \alpha(t) \exp (\beta t), \quad \forall 0 \leq t \leq T .
$$

\section{Main results}

In this section, we give criteria of average consensus for system (6).

\subsection{Average consensus in $p$ th moment for the WSNs}

Theorem 1 Assume that there is a function $V(t, i, \delta) \in C^{2,1}\left(R_{+} \times S \times R^{n} ; R_{+}\right)$and positive constants $p, c_{1}, c_{2}, \lambda$ and $\eta$ such that

$$
\begin{aligned}
& c_{1}|\delta|^{p} \leq V(t, i, \delta) \leq c_{2}|\delta|^{p}, \\
& \mathcal{L} V(t, i, \delta) \leq-\lambda|\delta|^{p}+\eta
\end{aligned}
$$

for all $t \geq 0 . i \in S$ and $\delta \in R^{n}$. Then (7) is exponential stable in pth moment.

Proof For the function $V(t, i, \delta)$, applying Lemma 1 and using the above conditions, we obtain that

$$
\begin{aligned}
c_{1} \mathbb{E}|\delta|^{p} & \leq \mathbb{E} V\left(0, r_{0}, \xi(0)\right)+\mathbb{E} \int_{0}^{t} \mathcal{L} V(s, r(s), \delta(s)) d s \\
& \leq \mathbb{E} V\left(0, r_{0}, \xi(0)\right)+\mathbb{E} \int_{0}^{t}\left(-\lambda|\delta|^{p}+\eta\right) d s .
\end{aligned}
$$

So,

$$
\begin{aligned}
\mathbb{E}|\delta|^{p} & \leq \frac{\mathbb{E} V\left(0, r_{0}, \xi(0)\right)}{c_{1}}+\int_{0}^{t} \frac{-\lambda \mathbb{E}|\delta|^{p}+\eta}{c_{1}} d s \\
& \leq c(t)+\int_{0}^{t} v \mathbb{E}|\delta|^{p} d s,
\end{aligned}
$$


where

$$
\begin{aligned}
& c(t)=\frac{\mathbb{E} V\left(0, r_{0}, \xi(0)\right)+\eta t}{c_{1}} \rightarrow \infty, \\
& \nu=-\frac{\lambda}{c_{1}} .
\end{aligned}
$$

It can be seen that $c(t)>0$ and $v<0$. By using Gronwall's inequality, we have

$$
\mathbb{E}|\delta|^{p} \leq c(t) \exp (v t)
$$

Therefore

$$
\limsup _{t \rightarrow \infty} \frac{1}{t} \log \left(\mathbb{E}|\delta(t, \xi)|^{p}\right) \leq v<0 .
$$

Thus (7) is exponential stable in $p$ th moment. This completes the proof.

Now we give a criterion of average consensus in $p$ th moment for system (6).

Theorem 2 Assume that for each $i \in S$, there is a pair of real numbers $\alpha>0$ and $\beta_{i}$ such that

$$
\delta^{T} f(\delta, t, i)+\frac{p-1}{2}|g(\delta, t, i)|^{2} \leq \alpha+\beta_{i}|\delta|^{2}
$$

for all $(\delta, t) \in R^{n} \times R_{+}$, where $p \geq 2$.

Assume also that

$$
M:=-\operatorname{diag}\left(p \beta_{1}, \ldots, p \beta_{N}\right)-\Gamma
$$

is a nonsingular $M$-matrix, in this case,

$$
\left(q_{1}, \ldots, q_{n}\right)^{T}:=M^{-1} \overrightarrow{1} \gg 0,
$$

where $\overrightarrow{1}=(1, \ldots, 1)^{T}$. Then system (6) reaches average consensus in $p$ th moment.

Proof We can see that

$$
\begin{array}{rl}
\delta^{T} & f(\delta, t, i)+\frac{p-1}{2}|g(\delta, t, i)|^{2} \\
& =-\delta^{T} L_{i} \delta+\frac{p-1}{2}\left|(I-J) G_{i}\right|^{2} \\
& \leq\left(\frac{p-1}{2}|(I-J)|^{2} H-\lambda_{\min }\left(L_{i}\right)\right)|\delta|^{2} \\
& \leq \alpha+\beta_{i}|\delta|^{2},
\end{array}
$$

where $\alpha \in R^{+}$and $\beta_{i}=\frac{p-1}{2}|(I-J)|^{2} H-\lambda_{\min }\left(L_{i}\right)$. 
Define the function $V: R_{+} \times S \times R^{n} \rightarrow R_{+}$by

$$
V(\delta, t, i)=q_{i}|\delta|^{p} .
$$

Clearly, $V$ obeys Theorem 1 with $c_{1}=\min _{i \in S} q_{i}$ and $c_{2}=\max _{i \in S} q_{i}$, and $c_{1}|\delta|^{p} \leq V(\delta, t, i) \leq$ $c_{2}|\delta|^{p}$.

We compute the operator $\mathcal{L} V$ as follows:

$$
\begin{aligned}
\mathcal{L} V(\delta, t, i)= & p q_{i}|\delta|^{p-2} \delta^{T} f(\delta, t, i)+\frac{1}{2} p q_{i}|\delta|^{p-2}|g(\delta, t, i)|^{2} \\
& +\frac{1}{2} p(p-2) q_{i}|\delta|^{p-4}\left|\delta^{T} g(\delta, t, i)\right|^{2}+\sum_{j=1}^{N} \gamma_{i j} q_{j}|\delta|^{p} \\
= & p q_{i}|\delta|^{p-2}\left(\delta^{T} f+\frac{p-1}{2}|g|^{2}\right)+\sum_{j=1}^{N} \gamma_{i j} q_{j}|\delta|^{p} \\
\leq & p q_{i}|\delta|^{p-2}\left(\beta_{i}|\delta|^{2}+\alpha\right)+\sum_{j=1}^{N} \gamma_{i j} q_{j}|\delta|^{p} \\
\leq & \left(p \beta_{i} q_{i}+\sum_{j=1}^{N} \gamma_{i j} q_{j}\right)|\delta|^{p}+\alpha p q_{i}|\delta|^{p-2} \\
= & -|\delta|^{p}+\alpha p q_{i}|\delta|^{p-2} .
\end{aligned}
$$

By Young's inequality,

$$
\begin{aligned}
\alpha p q_{i}|\delta|^{p-2} & =\alpha p q_{i} 2^{(p-2) / p}\left(\frac{1}{2}|\delta|^{p}\right)^{(p-2) / p} \\
& =\left[\left(\alpha p q_{i}\right)^{p / 2} 2^{(p-2) / 2}\right]^{2 / p}\left(\frac{1}{2}|\delta|^{p}\right)^{(p-2) / p} \\
& \leq \frac{2}{p}\left(\alpha p q_{i}\right)^{p / 2} 2^{(p-2) / 2}+\frac{(p-2)}{2 p}|\delta|^{p}
\end{aligned}
$$

Substituting this into (17) gives

$$
\mathcal{L} V(t, i, \delta) \leq-\frac{p+1}{p}|\delta|^{p}+\frac{2}{p}\left(\alpha p q_{i}\right)^{p / 2} 2^{(p-2) / 2} .
$$

Let

$$
\begin{aligned}
& \lambda=\frac{p+1}{p} \\
& \eta=\max _{i \in S}\left[\frac{2}{p}\left(\alpha p q_{i}\right)^{p / 2} 2^{(p-2) / 2}\right]=\frac{2}{p}\left(\alpha p c_{2}\right)^{p / 2} 2^{(p-2) / 2} .
\end{aligned}
$$

We can see that $\lambda, \eta$ are constants, so by Theorem 1 ,

$$
\limsup _{t \rightarrow \infty} \frac{1}{t} \log \left(\mathbb{E}|\delta(t, i, \xi)|^{p}\right) \leq v<0 .
$$


It means that

$$
\limsup _{t \rightarrow \infty} \mathbb{E}|\delta(t, i, \xi)|^{p} \leq \exp (v t) \stackrel{t \rightarrow \infty}{\longrightarrow} 0
$$

So system (6) reaches the average consensus in $p$ th moment. This completes the proof.

Remark 1 In Theorem 2, we can easily check the condition obtained by using the new method if the dynamic system is given and the positive constant $m$ is well chosen. To the best of our knowledge, the method combining the Lyapunov function and the $M$-matrix in this paper is rarely used in the researching area of the average consensus in $p$ th moment for WSNs with Markovian switching topology and stochastic noises.

Corollary 1 If the functions $f$, $g$ satisfy

$$
\begin{aligned}
& \delta^{T} f \leq k\left(\alpha+\beta_{i}|\delta|^{2}\right), \\
& \frac{p-1}{2}|g|^{2} \leq(1-k)\left(\alpha+\beta_{i}|\delta|^{2}\right),
\end{aligned}
$$

where $0<k<1, k \in \mathbb{R}$, then we get

$$
\delta^{T} f(\delta, t, i)+\frac{p-1}{2}|g(\delta, t, i)|^{2} \leq \alpha+\beta_{i}|\delta|^{2} .
$$

Following Theorem 2, system (6) reaches consensus in pth moment.

\subsection{The stochastic average consensus in the large for the WSNs under $\Gamma$ is not precisely known}

In the real communication networks, the part of the elements in the desired transition probabilities matrix are difficult to obtain. Therefore, it is important and necessary, from control perspectives, to further study the WSNs with partly unknown transition probabilities.

Consequently, in this part, we consider stochastic average consensus in the large for the system, even though the transition jump rates matrix $\Gamma=\left(\gamma_{i j}\right)_{N \times N}$ is not precisely known.

Theorem 3 Assume that there exist functions $V \in C^{2}\left(R^{n} \times S ; R_{+}\right)$, positive constants $\alpha_{1}, \alpha_{2}$ and for each $i \in S$, there exist a real number $\lambda>0$ and symmetric positive-definite matrices $P_{i}$ such that

(i)

$$
\alpha_{1}|\delta(t)|^{2} \leq V(\delta, i) \leq \alpha_{2}|\delta(t)|^{2}, \quad \forall i \in S
$$

(ii)

$$
2 \delta^{T} P_{i} f(\delta, i)+\operatorname{trace}\left(g^{T}(\delta, i) P_{i} g(\delta, i)\right) \leq-\lambda|\delta|^{2} ;
$$

(iii)

$$
V(\delta, i) \leq \frac{\alpha_{2}}{\alpha_{1}} V(\delta, j), \quad \forall i, j \in S
$$


(iv)

$$
1<\frac{\alpha_{2}}{\alpha_{1}}<\frac{\lambda+\tilde{q}}{\bar{q}},
$$

then the system reaches stochastic average consensus in the large.

Proof Define $V(\delta, i)=\delta^{T} P_{i} \delta$ for all $i \in S$. Let $\alpha_{1}=\min \left\{\lambda_{\min }\left(P_{i}\right): i \in S\right\}, \alpha_{2}=\max \left\{\lambda_{\max }\left(P_{i}\right)\right.$ : $i \in S\}$. Obviously,

$$
\alpha_{1}|\delta(t)|^{2} \leq V(\delta, i) \leq \alpha_{2}|\delta(t)|^{2}
$$

Also we can see that

$$
\begin{aligned}
\tilde{\mathcal{L}} V(\delta, i) & =2 \delta^{T} P_{i} f(\delta, i)+\operatorname{trace}\left(g^{T}(\delta, i) P_{i} g(\delta, i)\right) \\
& =-2 \delta^{T} P_{i} L_{i} \delta+\operatorname{trace}\left(g^{T}(\delta, i) P_{i} g(\delta, i)\right) \\
& \leq-2 \lambda_{\max }\left(P_{i}\right) \lambda_{\min }\left(L_{i}\right)|\delta|^{2}+H \lambda_{\max }\left(P_{i}\right)|\delta|^{2} \\
& =\left(-2 \lambda_{\min }\left(L_{i}\right)+H\right) \lambda_{\max }\left(P_{i}\right)|\delta|^{2} .
\end{aligned}
$$

Let $\lambda_{i}=\left(-2 \lambda_{\min }\left(L_{i}\right)+H\right) \lambda_{\max }\left(P_{i}\right)$ and $\lambda=\min \left\{\lambda_{i}: i \in S\right\}$, we have $\tilde{\mathcal{L}} V(\delta, i)<-\lambda_{i} V(\delta, i)<$ $-\lambda V(\delta, i)$.

On the other hand,

$$
V(\delta, i) \leq \frac{\alpha_{2}}{\alpha_{1}} V(\delta, j), \quad \forall i, j \in S
$$

Obviously, $1<u=\frac{\alpha_{2}}{\alpha_{1}}=\max \left\{\frac{\lambda_{\max }\left(P_{i}\right)}{\lambda_{\min }\left(P_{j}\right)}: i, j \in S\right\}<\frac{\lambda+\tilde{q}}{\bar{q}}$.

Consequently, according to Theorem 3.1 in [12], it is obtained that error system (7) is stochastic stable and

$$
\mathbb{P}\left\{\lim _{t \rightarrow \infty} \delta\left(t ; t_{0}, \delta_{0}, i\right)=0\right\}=1
$$

It means that system (6) reaches stochastic average consensus in the large. This completes the proof.

Remark 2 In contrast with the case that the transition probabilities of the underlying Markov chain are assumed to be completely known, the circumstance that transition jump rates matrix $\Gamma$ is not precisely known for the equation (7) is more general in the real control systems.

Remark 3 There are several disadvantages associated with the result derived in Theorem 3. (1) Hypothesis (22), it may reduce the set of systems to which the results in this section can be used. (2) The constructive method for choosing the best Lyapunov functions is currently unknown. 


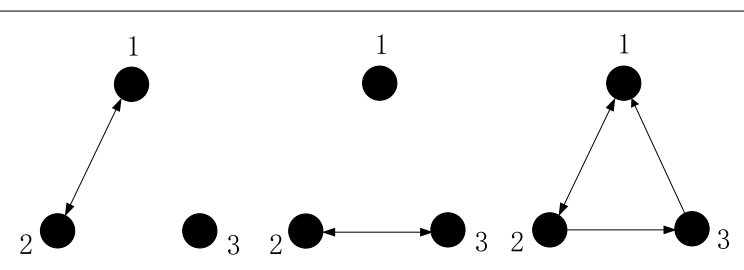

Figure 1 The topologies of the WSN in states 1, 2, 3.

\section{Numerical examples}

In this section, we give two examples to examine the average consensus of system (6).

Consider a WSN composed of three sensors in which each dynamic state of the sensor is $\dot{x}_{i}=u_{i}$, where $i=1,2,3$ (see the topologies in Figure 1), the initial state is $X(0)=(3,-2,4)^{T}$, the switching topology of the three sensors is determined by the Markov chain $r(t)$ whose state space is $S=\{1,2,3\}$. The related topology graph is $\mathcal{G}(i)=\{\mathcal{V}(i), \mathcal{E}(i), \mathcal{A}(i)\}$, the adjacent matrices are:

$$
\begin{array}{ll}
\mathcal{A}(1)=\left(\begin{array}{lll}
0 & 1 & 0 \\
1 & 0 & 0 \\
0 & 0 & 0
\end{array}\right), & \\
\mathcal{A}(2)=\left(\begin{array}{lll}
0 & 0 & 0 \\
0 & 0 & 1 \\
0 & 1 & 0
\end{array}\right), & \mathcal{A}(3)=\left(\begin{array}{ccc}
0 & 0.5 & 0 \\
1 & 0 & 0 \\
0 & 0.5 & 0
\end{array}\right) .
\end{array}
$$

The related Laplacian matrices are:

$$
\begin{aligned}
\mathcal{L}(1)=\left(\begin{array}{ccc}
1 & -1 & 0 \\
-1 & 1 & 0 \\
0 & 0 & 0
\end{array}\right), & \\
\mathcal{L}(2)=\left(\begin{array}{ccc}
0 & 0 & 0 \\
0 & 1 & -1 \\
0 & -1 & 1
\end{array}\right), & \mathcal{L}(3)=\left(\begin{array}{ccc}
0.5 & -0.5 & 0 \\
-1 & 1 & 0 \\
0 & -0.5 & 0.5
\end{array}\right) .
\end{aligned}
$$

Example 1 Suppose that the Markovian transition matrix is

$$
\Gamma=\left(\begin{array}{ccc}
-2 & 1 & 1 \\
1 & -2 & 1 \\
1 & 1 & -2
\end{array}\right) .
$$

We let $p=5, \alpha=2.1|\delta|^{2}, \beta_{i}=1, k=\frac{1}{2},|g|=0.15|\delta|$, it can be checked that assumptions (A1), (A2), and the conditions of Corollary 1 are satisfied and the matrix $M$ is a nonsingular $M$-matrix. So the WSN reaches average consensus by Corollary 1.

We can draw the dynamic curve of the states of the sensors by Matlab as Figure 2. It shows us that the three sensor nodes reach the average consensus in 5th moment. 


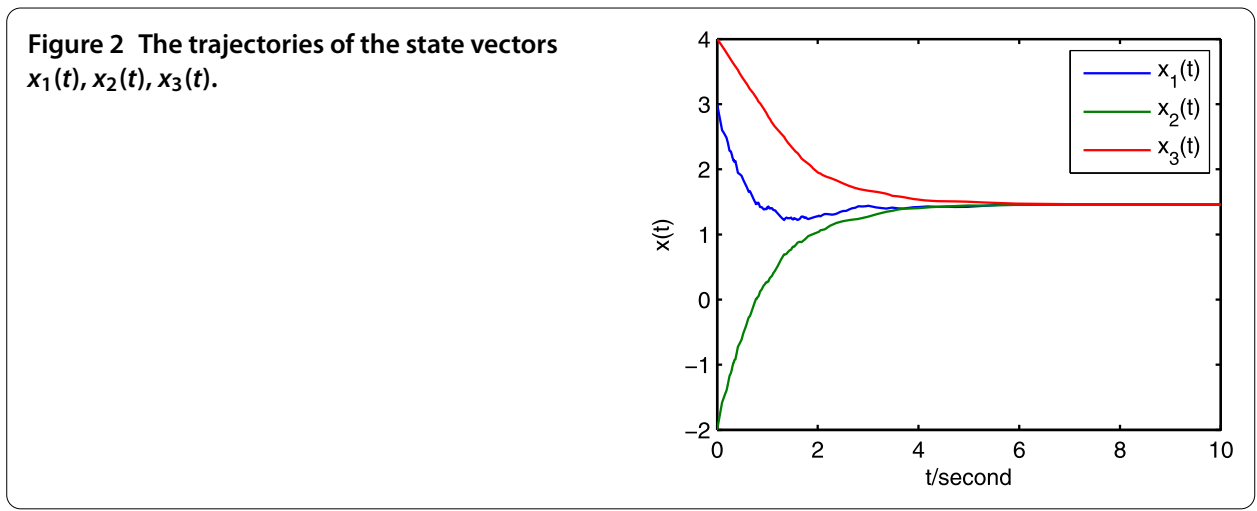

Figure 3 The trajectories of the state vectors $x_{1}(t), x_{2}(t), x_{3}(t)$.

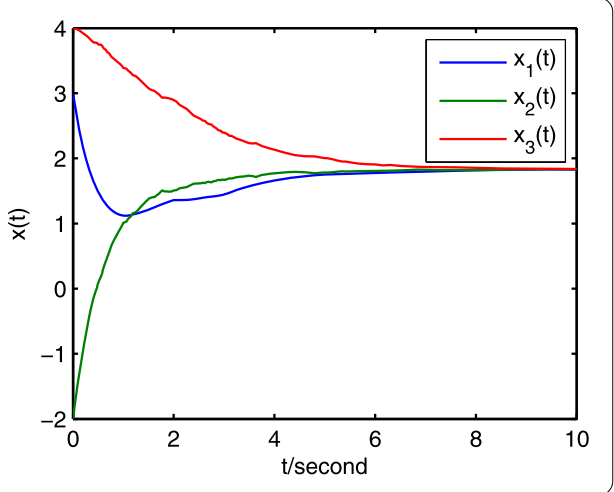

Example 2 Suppose that the Markovian transition matrix with partly unknown jump rates is

$$
\Gamma=\left(\begin{array}{ccc}
-a & b & 0 \\
1 & -2 & 1 \\
0 & 1 & -1
\end{array}\right)
$$

where $a, b>0$ denotes the unknown transition jump rates subject to $a \leq 2, b \leq 1$, respectively. We can see that $\tilde{q}=2, \bar{q}=1$.

Letting $|g|=0.25|\delta|$ and according to Theorem 3, we can draw the dynamic curve of the states of the sensors by Matlab as Figure 3. It shows us that the three sensor nodes reach stochastic average consensus in the large.

\section{Conclusions}

In this paper, we have dealt with the problem of average consensus in $p$ th moment of the WSNs. By using the Lyapunov function and the $M$-matrix approach, we have obtained several sufficient conditions to ensure the average consensus in $p$ th moment for WSNs with Markovian switching topology. Also, a sufficient condition for the stochastic average consensus in the large for the WSNs with partly known transition jump rates matrix $\Gamma$ is derived. There are many other topics such as the time-delay cases and the adaptive control of the WSN's consensus in $p$ th moment, etc. worth investigating. 


\section{Authors' contributions}

WZ and $\mathrm{CJ}$ carried out the main part of this manuscript. WZ participated in the discussion and corrected the main theorem. All authors read and approved the final manuscript.

\section{Acknowledgements}

This work is supported by the National Natural Science Foundation of China (61075060), the Innovation Program of Shanghai Municipal Education Commission (12zz064,13zz050), the Key Foundation Project of Shanghai (12JC1400400) and the Natural Science Foundation of Shanghai (12ZR1440200).

Received: 6 June 2013 Accepted: 5 November 2013 Published: 29 Nov 2013

\section{References}

1. Fax, JA, Murry, RM: Information flow and cooperative control of vehicle formations. IEEE Trans. Autom. Control 49, 1465-1476 (2004)

2. Olfati-Saber, R: Distributed Kalman filter with embedded consensus filters. In: Proceedings of the 44th IEEE Conference on Decision and Control, and the European Control Conference, Seville, Spain, pp. $8179-8184$ (2005)

3. Lynch, N: Distributed Algorithms. Morgan Kaufmann, San Matero (1996)

4. Olfati-Saber, R, Murray, RM: Consensus problem in networks of agents with switching topology and time-delays. IEEE Trans. Autom. Control 49, 1520-1533 (2004)

5. Kingston, DB, Beard, RW: Discrete-time average-consensus under switching network topologies. In: Proceedings of American Control Conference, Minneapolis, pp. 3551-3556 (2006)

6. Xiao, L, Boyd, S: Fast linear iterations for distributed averaging. Syst. Control Lett. 53, 65-78 (2004)

7. Zhang, Q, Zhang, J: Distributed consensus of continuous-time multi-agent systems with Markovian switching topologies and stochastic communication noises. J. Syst. Sci. Math. Sci. 31, 1097-1110 (2011)

8. Zhou, WN, Tong, DB, Gao, Y, Ji, C, Su, HY: Mode and delay-dependent adaptive exponential synchronization in $p$ th moment for stochastic delayed neural networks with Markovian switching. IEEE Trans. Neural Netw. Learn. Syst. 23, 662-668 (2012)

9. Zhou, WN, Mou, JP, Wang, TB, Ji, C, Fang, JA: Target-synchronization of the distributed wireless sensor networks under the same sleeping-awaking method. J. Franklin Inst. 349, 2004-2018 (2012)

10. Zhang, LX, Boukas, EK: Stability and stabilization of Markovian jump linear systems with partly unknown transition probability. Automatica 45, 463-468 (2009)

11. Zhang, LX, Lam, J: Necessary and sufficient conditions for analysis and synthesis of Markov jump linear systems with incomplete transition descriptions. IEEE Trans. Autom. Control 55, 1695-1701 (2010)

12. Zhu, F, Han, Z, Zhang, J: Stability analysis of stochastic differential equations with Markovian switching. Syst. Control Lett. 61, 1209-1214 (2012)

13. Mao, X, Yuan, C: Stochastic Differential Equations with Markovian Switching. Imperial College Press, London (2006)

14. Huang, C, Cao, J: On pth moment exponential stability of stochastic Cohen-Grossberg neural networks with time varying delays. Neurocomputing 73, 986-990 (2010)

15. Wang, X, Guo, Q, Xu, D: Exponential p-stability of impulsive stochastic Cohen-Grossberg neural networks with mixed delays. Math. Comput. Simul. 79, 1698-1710 (2009)

16. $\mathrm{Xu}, \mathrm{L}, \mathrm{Xu}, \mathrm{D}$ : Exponential $p$-stability of impulsive stochastic neural networks with mixed delays. Chaos Solitons Fractals 41, 263-272 (2009)

17. Wang, Z, Liu, Y, Liu, G, Liu, X: A note on control of discrete-time stochastic systems with distributed delays and nonlinear disturbances. Automatica 46, 543-548 (2010)

18. Wang, Z, Liu, Y, Liu, L, Liu, X: Exponential stability of delayed recurrent neural networks with Markovian jumping parameters. Phys. Lett. A 356, 346-352 (2006)

19. Li, T, Zhang, JF: Mean square average consensus under measurement noises and fixed topologies: Necessary and sufficient conditions. Automatica 45, 1929-1936 (2009)

20. Chatterjee, D, Liberzon, D: On stability of randomly switched nonlinear systems. IEEE Trans. Autom. Control 52, 2390-2394 (2007)

21. Berman, A, Plemmons, RJ: Nonnegative Matrices in Mathematical Sciences. SIAM, Philadelphia (1987)

10.1186/1687-1847-2013-346

Cite this article as: Zhou et al.: Consensus for wireless sensor networks with Markovian switching topology and

stochastic communication noises. Advances in Difference Equations 2013, 2013:346 